\title{
Analysis of Local Communication Load in Shape Formation of a Distributed Morphogenetic Swarm Robotic System
}

\author{
Hongliang Guo, Yan Meng, and Yaochu Jin
}

\begin{abstract}
Morphogenesis is the biological process that governs self-organized spatial pattern formation of cells during the embryonic development of multi-cellular organisms. Inspired by this process, we have proposed a morphogenetic framework for pattern formation and boundary coverage in a distributed swarm robotic system. The framework is based only on local communications among robots and will set up a local coordinate system. This paper focuses on the theoretical and empirical analysis of the framework regarding two aspects, namely, local communication load among the robots, and system performance such as system convergence time and average travel distance of robots for several pattern formation tasks. Results show that the proposed framework is efficient and scalable for self-organizing distributed swarm robotic systems with reasonable local communication load among robots.

Index Terms - self-organization, self-adaptation, swarm robots, morphogenesis, gene regulatory networks, pattern formation, and local communication
\end{abstract}

\section{INTRODUCTION}

$S_{n}$ warm robotics focuses on the control of large-scale multi-robot systems with limited communication and sensing capabilities. Compared to monolithic systems, swarm robotic systems have advantages such as robustness and adaptability, particularly under uncertain environments. Examples of applications of swarm robotic systems include foraging [12], box-pushing [14], aggregation and segregation [13], shape formation [2], cooperative mapping [22], soccer tournaments [21], site preparation [17], sorting [9] and collective construction [20].

Since centralized algorithms are vulnerable to system failures and dynamic environments, and cannot scale well to large systems, distributed control algorithms are more desirable for large-scale multi-robot systems. However, designing distributed and self-adaptive algorithms for swarm robotic systems is nontrivial. It is difficult, if not impossible, to predict an accurate emerging behavior from local interaction rules in a distributed control system [16].

To design a distributed multi-robot system and yet to have predictable global behavior, we turned our attention to biological systems for inspirations. Biological systems, from macroscopic swarm systems of social insects to microscopic

H. Guo and Yan Meng are with the Department of Electrical and Computer Engineering, Stevens Institute of Technology, Hoboken, NJ 07670, USA (Phone: 201-2165496, e-mail: hguo@stevens.edu, yan.meng@stevens.edu).

Yaochu Jin is with the Department of Computing, University of Surrey, Guildford, Surrey GU2 7TW, UK. (e-mail: yaochu.jin@surrey.ac.uk). cellular systems, can generate robust and complex emerging behaviors through local interactions subject to various kinds of uncertainties [11]. Inspired by the morphogenesis of biological organisms, a morphogenetic approach using a gene regulatory network (GRN) is proposed in our previous work for complex shape formation and boundary converge based on local communication among robots [7]. The basic idea of the framework is to treat each robot as a cell, and the interactions among the robots are modeled through the reaction-diffusion mechanism analogous to cell-cell signaling in multi-cellular organisms.

We have demonstrated that the proposed morphogenetic algorithm can deploy multiple robots to a specified shape uniformly only based on local information and local communication among robots. It is assumed that the description of the desired global pattern that robots will form is known by all the robots in advance. However, no global coordinate system is needed. Other global information of the system, such as the total number of robots in the system, the absolute position of the desired pattern, and the initial positions of all the robots are not known by robots either.

In this paper, we will mainly focus on the theoretical and empirical analysis of the GRN-based morphogenetic approach to multi-robot pattern formation with regard to local communication and formation performance analysis based on dynamic local coordinate systems and local neighborhood adaptation mechanism. We will first analyze how much local communication load has been added to the systems for the GRN-based approach without requiring a global coordinate system. Then a neighborhood adaptation mechanism will be proposed to ensure an even distribution of robots on the desired pattern.

The rest of the paper is organized as follows. Section II describes the proposed GRN-based model for self-organized multi-robot pattern formation, followed by the local communication load analysis in Section III. Case studies on two performance measures of the system, including convergence time and average travel distance of robots, for complex pattern formation tasks are presented in Section IV. This paper concludes by Section V.

\section{Gene Regulatory Network Model For SELF-ORgANIZED MULTI-ROBOT PATTERn FoRMATION}

\section{A. The Basic GRN Model}

From biological point of view, GRN is a network consisting of the interactions among DNA (genes), mRNA, 
morphogens, proteins and other chemicals in a cell. It plays a central role in understanding the organism development and natural evolution [1]. To understand the emergent morphology resulting from GRNs, many researchers in system biology have worked on the reconstruction of gene regulatory pathways through computational models. A large number of computational models for GRNs have been proposed [10, 5, 8, 15 and 19]. Among these models, ordinary or partial differential equations have been widely used.

In our previous work [7], we propose a GRN model that contains two types of artificial morphological proteins, $\mathrm{G}$ and $\mathrm{P}$, which guide the robots to move to the target shapes. In this GRN model, protein type $G$ consists of two proteins, which correspond to the $x$ and $y$ positions of a robot in a 2D environment, respectively. If a $3 \mathrm{D}$ shape is to be formed, three proteins of type $\mathrm{G}$ are needed to describe the position of the robot. Similarly, protein type P consists of two proteins for a 2D environment and three proteins for a 3D environment, which can be interpreted as the velocity of the robot. This is only a metaphor that tries to map protein concentrations in a cellular system to some physical properties in a swarm robotic system. Mathematically, as we will show later, the relationship between the concentration of protein $\mathrm{P}$ and the concentration of protein $\mathrm{G}$ in cells is more complex than that between position and velocity in robotic systems. The regulatory relationship between the artificial morphogen $\mathrm{m}$, protein type $\mathrm{P}$ and protein type $\mathrm{G}$ is depicted in Fig. 1.

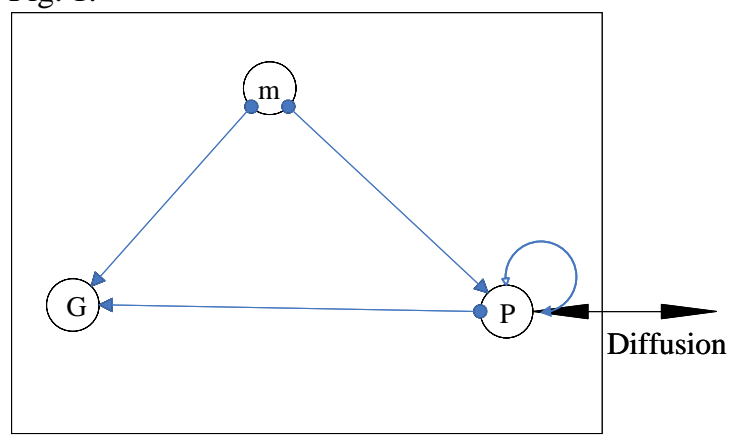

Fig.1.

The structure of the proposed GRN model: $\mathrm{m}$ is the predefined maternal morphogen, $\mathrm{G}$ and $\mathrm{P}$ are two types of morphological proteins.

From Fig. 1, we can see that morphogen m, which contains the target shape information, can regulate the production of both protein types $P$ and $G$ (indicated by the arrow). Protein type $P$ can also regulate the production of protein type $G$ in addition to an auto-regulation, i.e., it can influence its own production. Here, regulation can be either activation or repression.

The dynamics of the GRN model illustrated in Fig. 1 can be described by the following differential equations:

$$
\begin{gathered}
\frac{d \mathbf{g}_{i}}{d t}=-a \cdot \operatorname{grad}\left(\mathbf{g}_{i}\right)+\gamma \cdot \mathbf{p}_{i} \\
\frac{d \mathbf{p}_{i}}{d t}=-c \cdot \mathbf{p}_{i}+k \cdot f\left(\operatorname{grad}\left(\mathbf{g}_{\mathbf{i}}\right)\right)+b \cdot \mathbf{D}_{i}
\end{gathered}
$$

where $i$ is robot index. Note that the system is homogeneous. Each robot has the same GRN dynamic model. There are two protein products of type $G$ for formatting $2 \mathrm{D}$ shapes (representing the $\mathrm{x}$ - and $\mathrm{y}$ - position, respectively), and $\mathbf{g}_{i}$ is a vector of concentrations of protein $\mathrm{G}$ of robot $i$. Similarly, there are two protein products of type $\mathrm{P}$, and $\mathbf{p}_{i}$ is a vector of concentrations of protein $\mathrm{P}$ of robot $i$. The GRN model is similar in the case that $3 \mathrm{D}$ shapes are to be formed, except that there will be three protein products of type $G$ and $P$, respectively.

$\mathbf{D}_{i}$ denotes the summed protein concentration diffused from neighboring robots, which can be calculated by:

$$
\mathbf{D}_{i}=\sum_{j=1}^{N_{i}} \mathbf{D}_{i}^{j}
$$

where $N_{i}$ denotes the number of neighbors of robot $i$, and $\mathbf{D}_{i}^{j}$ is the protein concentration diffused from robot $j$ to robot $i$ and can be calculated by

$$
\mathbf{D}_{i}^{j}=\frac{\left(\mathbf{g}_{i}-\mathbf{g}_{j}\right)}{\left\|\mathbf{g}_{i}-\mathbf{g}_{j}\right\|}
$$

In real robotic systems, this protein diffusion procedure can be estimated by the local relative distances among the robots. In order for robot $i$ and robot $j$ to be neighbors, the distance between them must be smaller than the neighborhood size $d$. Here, the neighborhood size is adaptive. The neighborhood size $d$ is initialized as the average of the robot's bumper range and sensor range; it will be updated according to the neighborhood adaptation mechanism which will be described in Section III.B.

In Equ. (2), $\operatorname{grad}\left(\mathbf{g}_{\mathbf{i}}\right)$ is the maternal morphogen gradient at position $\mathbf{g}_{i}$ (as explained above, the concentration of protein type $\mathrm{G}$ in the cellular system represents the position of the robot). As depicted in Fig. 1, the concentration of both proteins $\mathrm{G}$ and $\mathrm{P}$ is influenced by the maternal morphogen gradient.

Note that the maternal morphogen gradient, $\operatorname{grad}\left(\mathbf{g}_{\mathbf{i}}\right)$, has slightly different impacts on the production of protein $\mathrm{G}$ and protein P. As we can see from Eqns. (1) and (2), $\operatorname{grad}\left(\mathbf{g}_{\mathbf{i}}\right)$ has a linear regulation on the concentration of protein $\mathrm{G}$, while its influence on concentration of protein $\mathrm{P}$ is further regulated by a nonlinear sigmoid function $f(z)$. The sigmoid function $f(z)$ in our model is defined as:

$$
f(z)=\frac{1-e^{-z}}{1+e^{-z}}
$$

There are five parameters in the GRN model described in Eqns. (1) and (2), namely, $a, c, k, b$, and $\gamma$. The following Theorem has been proved in our previous work [7].

Theorem: The dynamic system described by Eqns. (1) and (2) is globally asymptotically stable and the state vector $\mathbf{g}$ will converge to one point on the target curve $c(u)$ under the condition that $k \cdot \gamma \leq a \cdot c$ and $k, c, a, \gamma>0$. 


\section{B. Properties of the Extended GRN Model}

In the basic GRN-based algorithm for multi-robot pattern formation [6], it was assumed that robots know the absolute position of the target pattern as well as the initial position of themselves in a global coordinate system. In addition, if we want to deploy robots on the target shape evenly, each robot also needs to know the total number of robots in the system all the time. In this paper, we released these strong assumptions in an extended GRN-based model, where neither the absolute initial position of the robots nor the target shape position in the global coordinate system is required, only local distance detection from other robots are needed. Moreover, a mechanism for adapting the size of the neighborhood is also proposed, where only local communication among the robots is needed. In this way, even without knowing the total number of robots in the system, the robots can still autonomously adjust the neighborhood size to ensure an even coverage of the target shape.

\section{COMMUNICATION LOAD ANALYSIS}

In this extended model, the robots need to communicate with its neighbors to select a global reference robot and adapt the neighborhood size. We will analyze the system's communication load in the process of constructing a local coordinate system and adapting neighborhood size in this section.

\section{A. Communication Load Analysis for Construction of a Local Coordinate System}

First, the robots need to select a global reference robot to construct a local coordinate system. This selection mechanism consists of two main steps. First, all robots will compete locally, which results in a few local reference robots. Only those robots which have the largest number of robots in its neighborhood can be selected as local reference robots. Then, these local reference robots compete for the global reference through local communications. Since it is assumed that all the robots are connected to their neighbors in the system, if local reference robots are not neighbors, they can always communicate with each other through local communication relay using other non-local-reference-robots between them. Since the communication load would increase as the number of local reference robots increases, we use the number of local reference robots as a metric to measure the communication load during the construction the local coordinate system.

According to the definition of local reference robot, the number of local reference robots depends on the distribution of robots. Before we analyze the probabilistic distribution of the number of local reference robots, we make the following assumptions. (a)There are $n$ homogeneous robots in the system; (b) All the initialized robots are within a circle, and the radius of the circle is represented as $R$; (c) The neighborhood size of robots is determined by their sensing range. The robots are homogeneous so that they have the same sensing capability and the sensing range, which is denoted by $r$; (d) All the robots are randomly initialized, and they don't know the initial positions of other robots.

Based on these assumptions, the probabilistic distribution of the number of local reference robots can be estimated as follows:

1) For any robot $i$, it can have up to $n-1$ neighbors. The probability to be robot $i$ 's neighbor is:

$$
p=\frac{\pi r^{2}}{\pi R^{2}}=\frac{r^{2}}{R^{2}}
$$

2) From assumption d), we can say that the probability of robot $i$ having $k$ neighbors is a binomial distribution $K \sim B(n-1, p)$. Therefore, the probability mass function of this binomial distribution for robot $i$ can be defined as:

$$
p_{r}^{i}(K=k)=\left(\begin{array}{c}
n-1 \\
k
\end{array}\right) p^{k}(1-p)^{n-1-k}
$$

where $\left(\begin{array}{c}n-1 \\ k\end{array}\right)=\frac{(n-1) !}{k !(n-1-k) !}$, and $p$ is defined in Eqn. (6).

3) When robot $i$ has $k$ neighbors, the probability that robot $j$ has equal to or less than $k$ neighbors is:

$$
p_{r}^{j}(K \leq k)=\sum_{l=1}^{k}\left(\begin{array}{c}
n-1 \\
k
\end{array}\right) p^{l}(1-p)^{n-1-l}
$$

Note that robot $j$ can have $l$ neighbors with $1 \leq l \leq k$ because robot $j$ automatically has robot $i$ as its neighbor.

4) The probability that robot $i$ is a local reference robot is equal to the probability that all of its $k$ neighbors have equal to or less than $k$ neighbors, which is

$$
p_{i}=\sum_{k=1}^{n-1} p_{r}^{i}(K=k)\left[p_{r}^{j}(K \leq k)\right]^{k}
$$

5) According to assumption a), $n$ robots are homogeneous, each robot has a probability of $p_{i}$ to be the local reference robot, so the probability that there are $m$ local reference robot is:

$$
p_{m}=\left(\begin{array}{c}
n \\
m
\end{array}\right)\left(p_{i}\right)^{m}\left(1-p_{i}\right)^{n-m}
$$

Where the expectation of the number of local reference robots is $n p_{i}$ and the standard deviation is $\sqrt{n p_{i}\left(1-p_{i}\right)}$.

From Eqn. (10), it can be hypothesized that the final distribution of the local reference robots is a binomial distribution, which can be verified through the following simulations. First, we conducted 30000 experimental runs using 20 robots to form a unit circle, where the communication range of robots is setup as one-third of the whole area, the histogram of the number of the local reference robots in the experiments is shown in Fig. 2. Then, we use the Monte Carlo method to draw samples from a binomial distribution $B(20,0.19)$ for 30000 runs, the simulation results are shown in Fig. 3. 


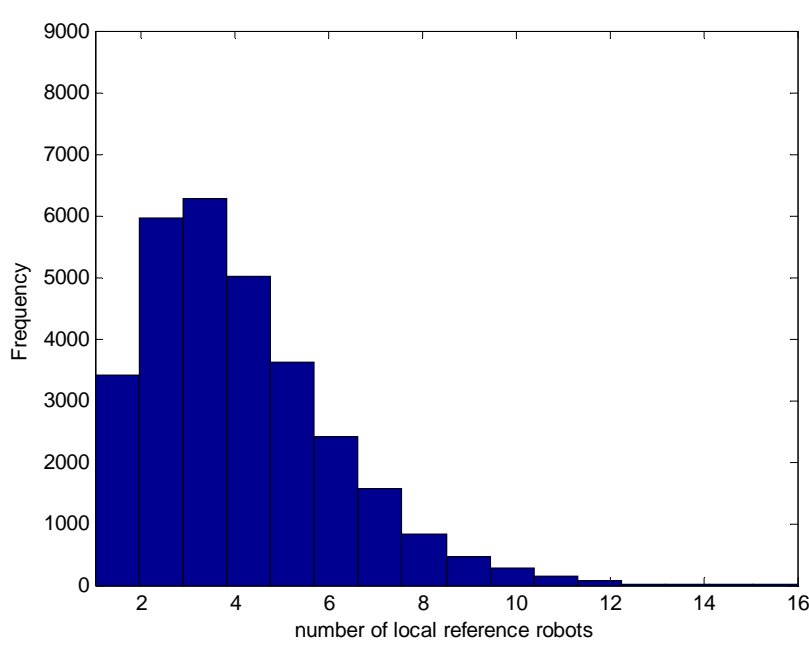

Fig. 2: Histogram of the number of local reference robots with a mean value of 3.81 and the standard deviation of 2.14.

From Fig. 2 and Fig. 3, we can see that the distribution of the number of local reference robots in Fig. 2 is similar to the binomial distribution drawn from the Monte Carlo method. Therefore, we can tentatively conclude that the distribution of the number of local reference robots is a binomial distribution as we have predicted in Eqn. (10). The total number of local reference robots is 3.81 in mean with the standard deviation of 2.14. In other words, the communication load is not heavy for this swarm robotic system.

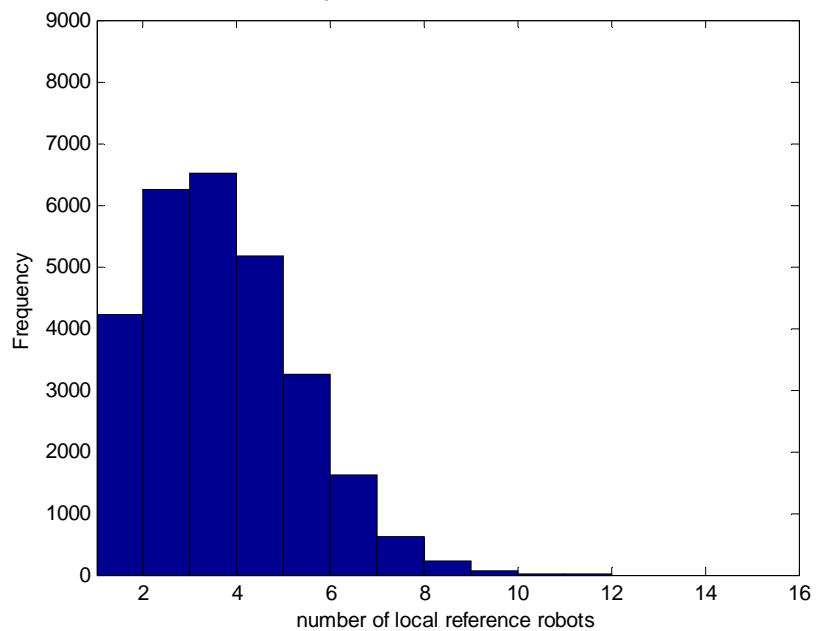

Fig. 3: Histogram of the number of local reference robots drawn from the Monte Carlo Method.

B. Communication Load Analysis for Neighborhood Size Adaptation

The major steps of the neighborhood size adaptation are listed as follows.

1) Initialization: $d \_\min$ and $d \_$max are initialized as the bumper range and sensor detection range of a robot, respectively, and the initial neighborhood size is estimated by

$$
d_{0}=\frac{d_{-} \min +d_{-} \max }{2}
$$

2) If $d_{0}$ is too small, we keep $d \_$max the same and update $d \_$min and $d_{0}$ as follows:

$$
\begin{aligned}
& d_{-} \min =d_{0} \\
& d_{0}=\frac{1}{2}\left(d_{0}+d_{-} \text {max }\right)
\end{aligned}
$$

3) If $d_{0}$ is too large, we keep $\$ d$ min $\$$ the same and update $\$ d \_m a x \$$ and $d_{0}$ as follows:

$$
\begin{aligned}
& d_{-} \max =d_{0} \\
& d_{0}=\frac{1}{2}\left(d_{0}+d_{-} \text {min }\right)
\end{aligned}
$$

4) Repeat step (2) or (3) until the optimal neighborhood size is found.

Details about how the robots know that the current neighborhood $d_{0}$ is smaller or larger than the expected optimal neighborhood $d^{*}$ are described in [7]. For simplicity, we suppose that

$$
\left|d_{0}-d^{*}\right| \leq \varepsilon .
$$

It means that the robots have found the optimal neighborhood and do not need to update $d_{0}$ anymore, where $\varepsilon$ is the threshold which makes the current neighborhood of a robot the expected one.

Now we need to analyze the communication load for the neighborhood adaptation mechanism. In the adaptation mechanism, we can see that communication among robots happens only when robots realize that they need to update $d_{0}$, therefore, we use the number of times that the robots need to update $d_{0}$ as the metric to measure the communication load.

The proposed neighborhood adaptation mechanism is a typical search algorithm. The largest number of times needed to find the optimal neighborhood is:

$$
n=\left[\log _{2}\left(\frac{d_{-} \max -d_{-} \min }{\varepsilon}\right)\right]_{-}
$$

where $[x]$ _denotes the biggest integer that is smaller than $x$.

The probability that robots need $k(0 \leq k \leq n)$ times to find the optimal neighborhood range is:

Case 1: If $k=0$, which means that $d^{*}$ is within the range of $\left[d_{0}-d^{*}, d_{0}+d^{*}\right]$, so the probability is

$$
P_{k}=\frac{2 \varepsilon}{d_{-} \max -d_{-} \min } \text {. }
$$

Case 2: If $0<k<n$, every time $d_{0}$ is updated and a new search is started, the search space will shrink by half. Thus, the probability at which $d^{*}$ is found this time will be twice that of the previous time, but this is a conditional probability given that $d^{*}$ has not been found in the previous $(k-1)$ times, so the probability will be:

$$
P_{k}=\left(1-\sum_{i=0}^{k-1} P_{i}\right) \cdot \frac{2^{k+1} \cdot \varepsilon}{d_{-} \max -d_{-} \min }
$$

Case 3: If $k=n, d^{*}$ has been found, so the probability is 
equal to the probability at which $d^{*}$ has not been found in the previous $n-1$ times:

$$
P_{k}=1-\sum_{i=0}^{k-1} P_{i}
$$

To make it clear, we will use an example to illustrate the number of needed times for a swarm robotic system to reach $d^{*}$.

Suppose that $d_{-} \min =2, d_{-} \max =200$, and $\varepsilon=0.5$, here we do not need the total number of robots, neither do we need to know the target pattern. We are just focusing on how many iteration of updates is needed for robots to achieve the optimal neighborhood size $d^{*}$. 30000 experimental runs have been conducted, where $d^{*}$ is set up as a random variable with a uniform distribution between $d_{-} \min$ and $d_{-} \max$. In this case study, the maximum number $n$ required for updates can be calculated by Equation (17) as:

$$
n=\left[\log _{2}\left(\frac{d_{-} \max -d_{-} \min }{\varepsilon}\right)\right]_{-}=8
$$

Fig. 4 shows the distribution of the number of updates needed. From Fig. 4, we can see that after 5 or 6 times of adjustments of $d_{0}$, the robots would reach the expected optimal neighborhood size. In other words, the communication load for this procedure is not heavy either.

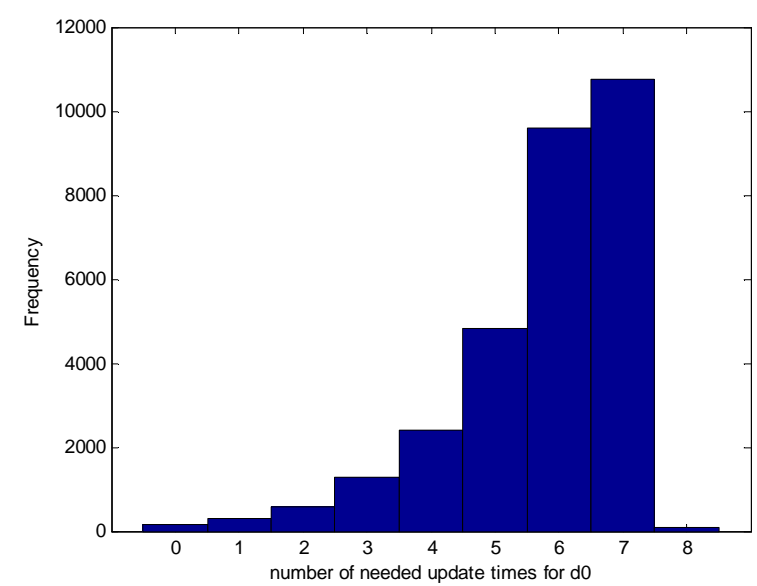

Fig. 4: Histogram of the number of needed number of updates to achieve the optimal neighborhood size. The mean value is 5.7576 and the standard deviation is 1.3903 .

\section{EXPERIMENTAL RESULTS}

In this section, we will demonstrate the performance of the GRN-based model using a local coordinate system and a neighborhood adaptation mechanism for multi-robot pattern formation. Two performance measures are considered, namely, the average travel distance of all robots and the convergence time of the slowest robot. The parameters $(a, c, k, b, \gamma)$ in the model are first optimized by minimizing those two performance measures simultaneously, which is a multi-objective optimization problem. To this end, NSGA-II [4], which is a popular evolutionary multi-objective algorithm, has been employed to achieve the Pareto-optimal solutions that trade off between the convergence time and the average travel distance. In this paper, we choose one Pareto-optimal solution that has a balanced performance on both metrics, whose parameters are: $k=52.12, c=77.84$, $b=239.81, a=9.96$ and $\gamma=4.67$.

The simulation configurations are as follow: (1) the simulation is implemented through Matlab 2007b, and the environment is assumed to be a 30x30 square field; (2) robots are initialized randomly in the environment; (3) we assume that the agents are holonomic so that they can have any instant moving direction.

\section{A. Case Study 1: Basic Shape Formation}

In this case study, we will measure the system performance, i.e., the convergence time and the average travel distance of the robots, by deploying a number of initially randomly distributed robots to a circle of a radius 5, but the central point of the circle is unknown in advance. First, a few local reference robots will emerge from the robot group, then, through local communications, the local reference robots will select a global reference robot to construct the local coordinate system and the desired shape will be formed based on this local coordinate system by following the GRN-based dynamics defined in Eqns. (1) and (2).

Fig. 5 shows a set of snapshots of the procedure of selecting the global reference robot and the deployment of robots to the target circle based on the selected reference robot. Fig. 6 shows the neighborhood adaptation process. In Fig. 6, we can see that the neighborhood (solid line) approaches the expected optimal neighborhood (dash solid line). Note that the expected optimal neighborhood can be calculated by dividing the perimeter of the desired curve by the number of robots. However, this information is unknown to the robots. Robots can only use the adaptation mechanism to adjust their neighborhood size.

We test the system performance for 5 robots, 10 robots, 15 robots and 20 robots, respectively. We run the simulation 100 times for each set of experiments, and then we calculate the mean and standard deviation of the convergence time and the average travel distance of robots, which are listed in Table I. From Table I, we can see that the convergence time and average travel distance of robots increase just slightly as the number of robots increases, which demonstrates that the proposed GRN-based algorithm with local communication is scalable to the number of robots. Note that the average travel distance of robots depends on the initial distribution (configuration) of the robots. If the multi-robot system is initialized as a cluster, a global reference robot would 'almost' represent the center of the cluster. In this case, we can conclude that the average travel distance of robots will not become much larger as the number of robots increases.

Then, we compared the system performance of this extended GRN-based model with local coordinate system and neighborhood size adaptation with that of the basic GRN-based model using a global coordinate system and some global information. In the basic GRN-based model [6], we designed a fixed pattern defined in a global coordinate 
system regardless of the robots' initial configuration and assume that all robots know the location of the target pattern. Table II lists the results of the basic GRN-based model for a circle formation task.

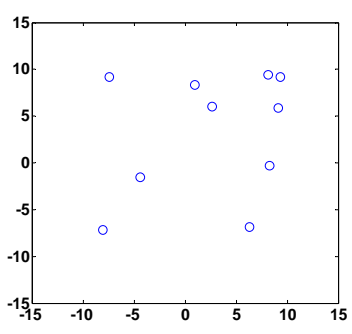

(a)

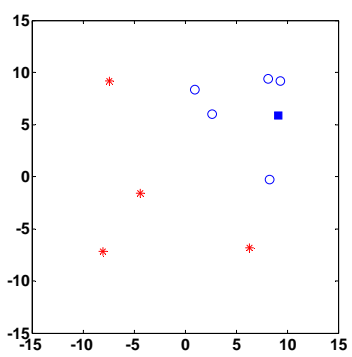

(c)

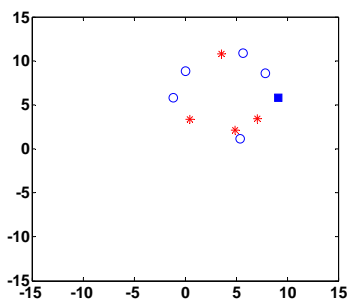

(e)

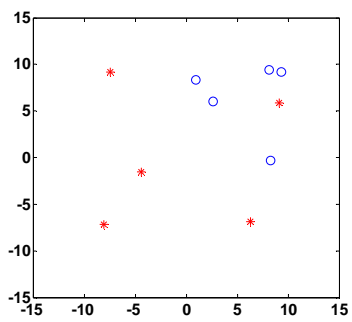

(b)

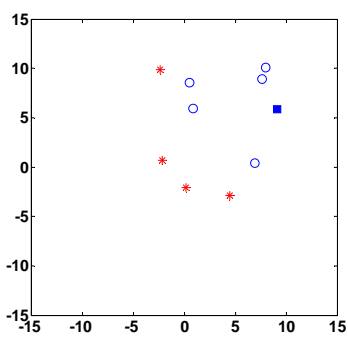

(d)

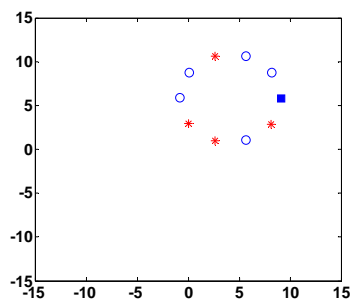

(f)
Fig. 5. (a) Initial distribution of 10 robots; (b) Local reference robots emerge from the multi-robot system (denoted by red stars); (c) The global reference robot is selected through series of local communications (denoted by a blue square); (d)(e) the circle formation process; (f) The robots form a circle.

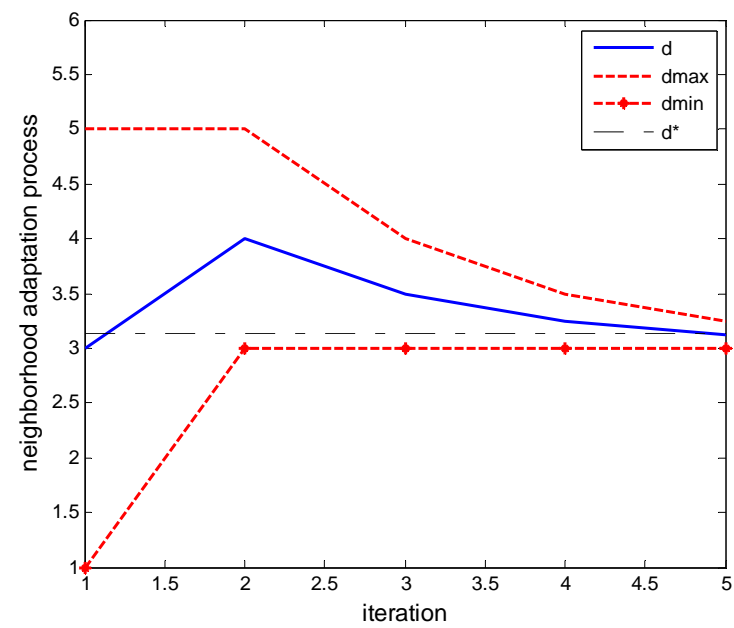

Fig. 6. Neighborhood adaptation process for 10 robots, where the circle radius is 5, $d \_\max$ is $5, d \_$min is 1 , and $\varepsilon$ is 0.05 .
TABLE I

SYSTEM PERFORMANCE STATISTICS FOR THE PROPOSED GRN MODEL

\begin{tabular}{lcccc}
\hline \hline \multicolumn{1}{c}{ Performance } & 5 robots & 10 robots & 15 robots & 20 robots \\
\hline $\begin{array}{l}\text { Convergence } \\
\text { time (mean) }\end{array}$ & 20.5672 & 21.9200 & 22.2800 & 23.4700 \\
$\begin{array}{l}\text { Convergence } \\
\text { time (std) }\end{array}$ & 1.9865 & 1.5328 & 1.9953 & 1.8116 \\
$\begin{array}{l}\text { Average travel } \\
\text { distance (mean) } \\
\begin{array}{l}\text { Average travel } \\
\text { distance (std) }\end{array}\end{array}$ & 10.4120 & 10.4615 & 10.7141 & 11.0314 \\
\hline \hline
\end{tabular}

TABLE II

SYSTEM PERFoRMANCE STATISTICS FOR THE GRN MODEL WiTH GLOBAL COORDINATE SYSTEM

\begin{tabular}{lcccc}
\hline \hline \multicolumn{1}{c}{ Performance } & 5 robots & 10 robots & 15 robots & 20 robots \\
\hline $\begin{array}{l}\text { Convergence } \\
\text { time (mean) }\end{array}$ & 20.0600 & 23.1000 & 24.5800 & 25.9800 \\
$\begin{array}{l}\text { Convergence } \\
\text { time (std) }\end{array}$ & 1.5296 & 1.7464 & 2.0210 & 2.3137 \\
$\begin{array}{l}\text { Average travel } \\
\text { distance (mean) }\end{array}$ & 13.9368 & 20.0838 & 20.3227 & 20.9574 \\
$\begin{array}{l}\text { Average travel } \\
\text { distance (std) }\end{array}$ & 3.1883 & 2.4941 & 1.8091 & 1.7668 \\
\hline \hline
\end{tabular}

Comparing the results in Table II with those in Table I, we can see that the algorithm using a local coordinate system outperforms the one using a global coordinate system with respect to both convergence time and average travel distance.

\section{B. Case Study 2: Shape Transition}

In this case study, we will evaluate the performance of the improved GRN-based model with a local coordinate system when the desired target pattern changes. We have shown in our previous work [7] that the GRN model is capable of changing the formed patterns as long as the definition of morphogen is changed in robots' dynamics. In this paper, we will focus on the analyzing the statistical results of the average travel distance of robots and the system convergence time.

Suppose initially 20 robots have formed a circle in the environment, then the morphogen level is changed to let the robots to form a letter ' $R$ '. Letter ' $R$ ' is represented using the Non-Uniform Rational B-Spline (NURBS) [3][18], which is a mathematical model commonly used in computer graphics and design optimization for generating and representing curves and surfaces. NURBS can offer a common mathematical form for both analytic and free-form shapes, hence providing a high flexibility in designing shapes. Fig. 7 shows a set of snapshots of the shape transition process. Note that in this case study, we do not reselect a global reference robot to construct the local coordinate system, because a circle is isotropic and hence every robot can be used as the global reference robot.

The simulation was run 100 times for each set of experiment and Table III lists the statistical results with the mean values and stand deviations of the system performance, i.e., the convergence time and the average travel distance of robots. 


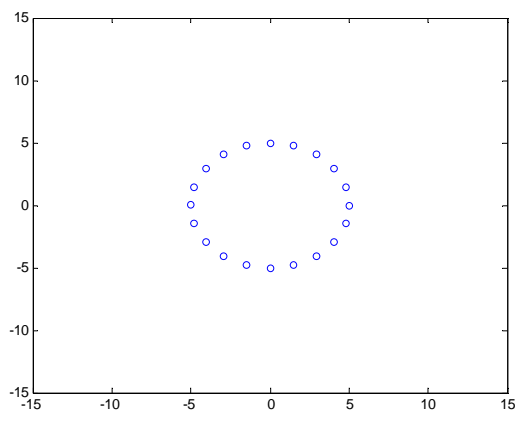

(a)

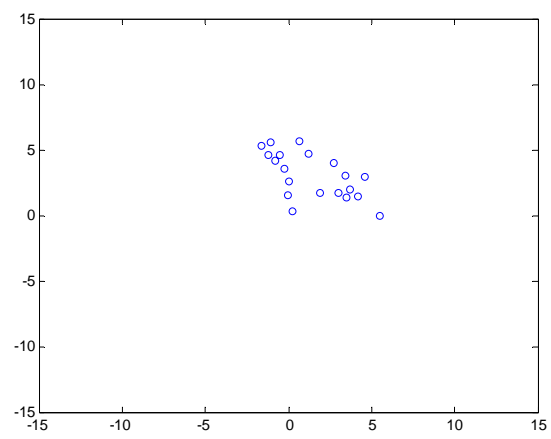

(b)

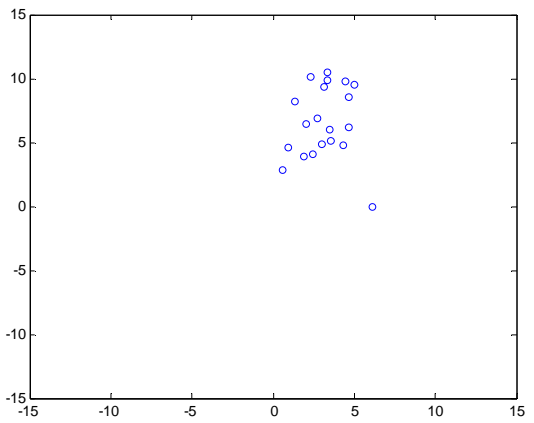

(c)

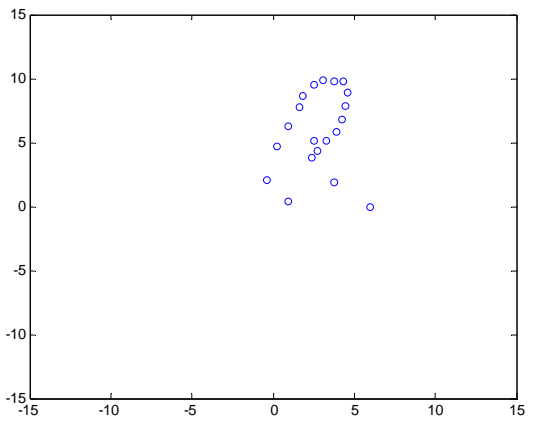

(d)

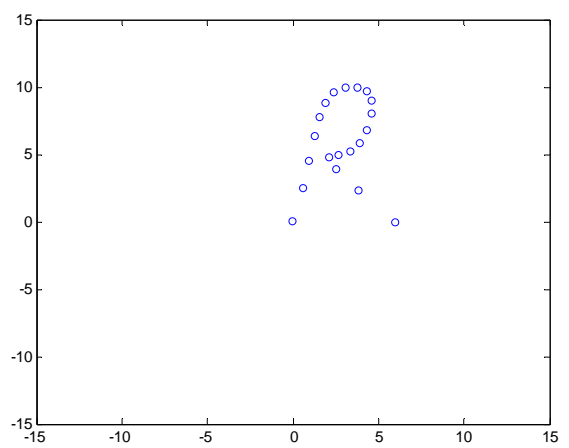

(e)

Fig. 7. (a) Phase one of the ' $R$ ' formation process, $t=0$; (b) Phase two of the ' $R$ ' formation process, $t=3$; (c) Phase three of the ' $R$ ' formation process, $t=10$; (d) Phase four of the ' $R$ ' formation process, $t=16$; (e) The robots form letter ' $R$ '.

TABLE III

SySTEM PERFoRMANCE METRICS OF THE GRN MODEL FOR SHAPE TRANSITION

\begin{tabular}{lcccc}
\hline \hline \multicolumn{1}{c}{ Performance } & 5 robots & 10 robots & 15 robots & 20 robots \\
\hline $\begin{array}{l}\text { Convergence } \\
\text { time (mean) }\end{array}$ & 21.0000 & 24.0000 & 24.0000 & 26.0000 \\
$\begin{array}{l}\text { Average travel } \\
\text { distance (mean) }\end{array}$ & 8.5297 & 9.99875 & 10.3963 & 10.7909 \\
\hline \hline
\end{tabular}

Since this case study focuses on the shape transition property, where the robots always start from the same initial distribution (previously formed shape), the standard deviation for performance measures is zero. Therefore, only the mean values of listed in Table III. From Table III, we can see that the average travel distance of robots and the convergence time do not increase much as the number of robots increases, which shows the scalability of the proposed GRN model.

When the target shape changes, the initially converged system described by Eqns. (1) and (2) becomes dynamic again. With the internal dynamics defined in Eqns. (1) and (2), each robot can automatically readjust its movement behaviors to reach another converged state of the systems.

\section{Case Study 3: A Proof-of-Concept Robot Experiment}

To further verify the feasibility of the extended GRN-based model in a distributed swarm robotic system, 6 e-puck robots have been used to implement the proposed algorithm to form a circle in an indoor environment. The snapshots of this proof-of-concept experiment are shown in Fig. 8. From Fig.8, it can be seen that the proposed extended GRN-based model can work efficiently with a local coordinate system and with neighborhood adaptation mechanism in a distributed manner. 


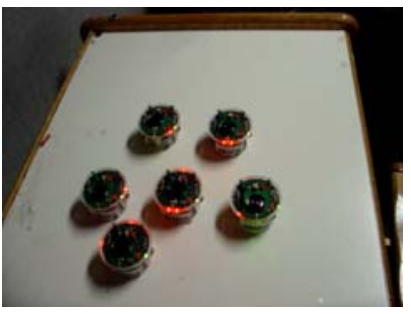

(a)

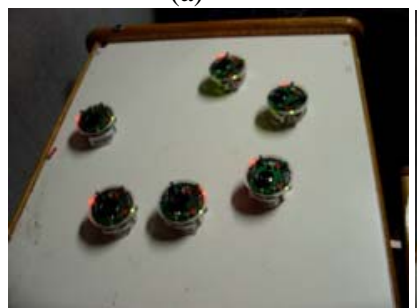

(c)

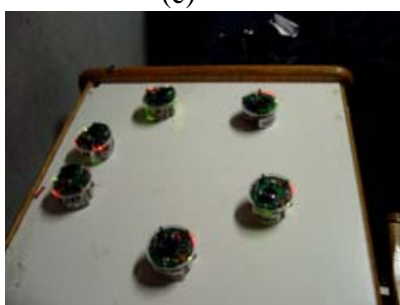

(e)

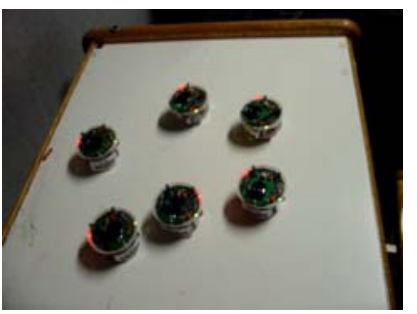

(b)

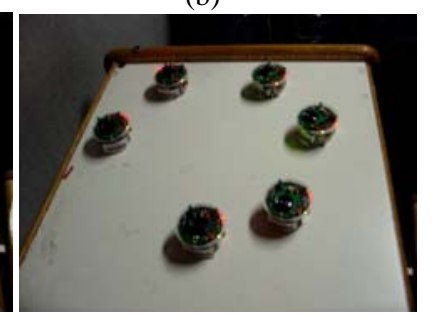

(d)

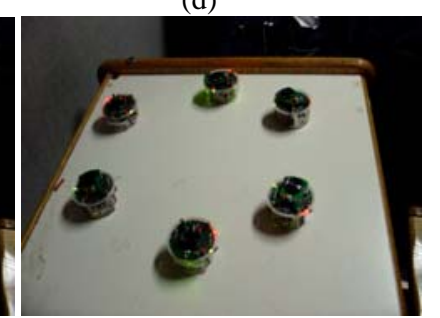

(f)
Fig. 8. Snapshots of the experiment. (a) The initial distribution, the center robot is selected as the reference robot and the relative distances of other robots can be detected by onboard sensors. (b) (c) (d) The pattern (a circle) formation process, after the formation, there is a vacant place in (d) indicating that the neighborhood is shorter than expected. (e) The pattern formation after neighborhood adjustment, this time two robots are stuck together, which means that the neighborhood is larger than expected. (f) The pattern formation after another phase of neighborhood adjustment. Robots are distributed uniformly on the circle.

\section{CONCLUSION AND FUTURE WORKS}

In this paper, we have studied the communication load and system performance of a distributed GRN-based approach to self-organizing multi-robot systems for formation control. First, we presented probabilistic analysis and empirical verification of the communication load of the extended GRN-based model for constructing a local coordinate system. Second, we presented the needed communication load for neighborhood adaptation. Third, extensive simulations have been conducted to examine the performances of the extended GRN-based model using a local coordinate system and a neighborhood adaptation mechanism. Compared to the basic GRN-based model with a global coordinate system and global information, the extended GRN-based model shows better performance. In addition, we demonstrate that both the communication load and system performance (average travel distance and convergence time) are fairly scalable to the number of robots in the system.

In the future, we will investigate robustness of the proposed model considering various system noises. One of the major limitation of the current model is that the target patterns have to be predefined. In the future, we will investigate a hierarchical model which can generate the target pattern dynamically through sensor information to adapt to environmental changes.

\section{REFERENCES}

[1] U. Alon, An Introduction to Systems Biology: Design Principles of Biological Circuits. Chapman \& Hall/CRC, July 2006.

[2] T. Balch and R. C. Arkin, "Behavior-based Formation Control for Multi-robot Teams”, IEEE Trans. on Robotics and Automation, 1999.

[3] W. Bohm, G. Farin, and J. Kahmann. A survey of curve and surface methods in CAGD. Computer Aided Geometric Design, 1(1):1-60, 1984.

[4] K. Deb, A. Pratap, S. Agarwal, and T. Meyarivan. A Fast and Elitist Multiobjective Genetic Algorithm: NSGA-II. IEEE Transactions on Evolutionary Computation, Vol. 6, No. 2, 2002. pp.182-197.

[5] D. Endy and R. Brent. Modeling cellular behavior. Nature 409, 391-395. 2001.

[6] H. Guo, Y. Meng and Y. Jin, A Cellular Mechanism for Multi-Robot Construction via Evolutionary Multi-Objective Optimization of a Gene Regulatory Networks, BioSystems, 98(3):193-203, 2009.

[7] H. Guo, Y. Jin and Y. Meng: A Unified Framework for Self-Organized Multi-Robot Pattern Formation and Boundary Coverage Inspired from Morphogenesis, ACM Transactions on Adaptive and Autonomous Systems (TAAS), 2010 (submitted).

[8] J. Hasty, D. McMillen, F. Isaacs., and J.J. Collins, Computational studies of gene regulatory networks: In numero molecular biology, Nat. Rev. Genet. 2, 268-279. 2001.

[9] O.E. Holland and C. Melhuish, "Stigmergy, Self-Organization, and Sorting in Collective Robotics”, Artificial Life, Vol. 5, pp. 173-202, 1999.

[10] H. De Jong, Modeling and simulation of genetic regulatory systems: a literature review. Journal of Computational Biology, vol. 9, no. 1, pp. 67-103, 2002.

[11] K. Kelly, Out of Control - The New Biology of machines, Social Systems and Economic World. Basic Books, 1994.

[12] M.J.B. Krieger, J. B. Billeter, and L. Keller, “Ant-like Task Allocation and Recruitment in Cooperative Robots,” Nature, Vol. 406, 2000, pp. 992-995.

[13] A. Martinoli, A. J. Ijspeert, and F. Mondada, "Understanding Collective Aggregation Mechanisms: From Probabilistic Modeling to Experiments with Real Robots”, Robotics and Autonomous Systems, Vol. 29, pp. 51-63, 1999.

[14] M. J. Mataric, M. Nilsson, and K. T. Simsarian, "Cooperative Multi-robot Box-Pushing”, IEEE/RSJ International Conference on Intelligent Robots and Systems, 1995.

[15] H. McAdams, and A. Arkin. Simulation of prokaryotic genetic circuits. Ann. Rev. Biophys. Biomol. Struct. 27, 199-224. 1998.

[16] S. Nolfi and D. Floreano: Evolutionary Robotics: The Biology, Intelligence, Technology of Self-Organizing Machines, MIT Press, Cambridge, MA, 2000.

[17] C.A.C. Parker and H. Zhang, "Collective Robotic Site Preparation”. Adaptive Behavior. Vol.14, No. 1, 2006, pp. 5-19.

[18] L. Piegl. On NURBS: A survey. IEEE Computer Graphics and Applications, 11(1):55-71, Jan. 1991.

[19] P. Smolen, D.A. Baxter and J.H. Byrne, Modeling transcriptional control in gene networks: Methods, recent results, and future directions, Bull. Math. Biol. 62, 247-292. 2000.

[20] R. L. Stewart and R. A. Russell. “A Distributed Feedback Mechanism to Regulate Wall Construction by a Robotic Swarm”. Adaptive Behavior. 14(1):21-51, 2006.

[21] T. Weigel, J. S. Gutmann, m. Dietl, A. Kleiner, and B. Nebel, “CS Freiburg: Coordinating Robots for Successful Soccer Playing”, Special Issue on Advances in Multi-Robot Systems, T. Arai, E. Pagello, and L. E. Parker, Editors, IEEE Trans. on Robotics and Automation, Vol. 18, No.5, pp. 685-699, 2002.

[22] B. Yamauchi, "Decentralized Coordination for Multi-robot Exploration”, Robotics and Autonomous Systems, Vol. 29, No. 1, pp. 111-118, 1999. 